\title{
Estudio sobre la investigación en marketingy su importancia en los procesos de exportación: análisis bibliométrico y temático en Scopus*
}

\author{
WILLIAM A. MALPICA ZAPATA** \\ MELVA INÉS GÓMEZ CAICEDO*** \\ ÁLVARO VILLA MARTÍNEZ****
}

\footnotetext{
* Artículo de revisión derivado del proyecto de investigación “Estrategia de internacionalización apoyada en el marketing digital para organizaciones dedicadas a producir y comercializar bebidas orgánicas en Colombia”, desarrollado durante el 2020 por docentes de la Universidad Piloto de Colombia y la Fundación Universitaria Los Libertadores.

** Magíster en Dirección y Administración de Empresas. Universidad Piloto de Colombia, Bogotá, Colombia. E-mail: william-malpica@unipiloto.edu.co. ORCID: 0ooo-0oo2-0989-8465. Google Scholar: https://scholar.google.com/ citations? user $=$ CZxJTioAAAAJ\&hl=es.

*** Magíster en Dirección General. Fundación Universitaria Los Libertadores, Bogotá, Colombia. E-mail: migomezc@libertadores.edu.co. ORCID: 0000-0002-9020-0051. Google Scholar: https://scholar.google.com/citations?hl=es\&user=FTO4tnMAAAAJ. Scopus Author ID: https://www.scopus.com/authid/detail.uri?authorld=57224223294.

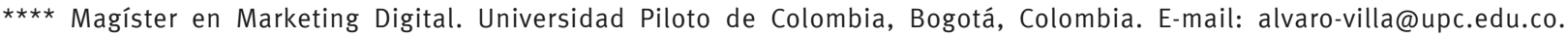
ORCID: 0000-0003-0102-4824. Google Scholar: https://scholar.google.es/citations?hl=es\&user=7cbWPQoAAAA).
} 
COMO CITAR ESTE ARTÍCULO

How to cite this article:

Malpica, W.A., Gómez, M.I. y Villa, Á. (2021). Estudio sobre la investigación en marketing y su importancia en los procesos de exportación: análisis bibliométrico y temático en Scopus. Revista Perspectiva Empresarial, 8(2), 91-103.

Recibido: 22 de junio de 2021 Aceptado: 08 de octubre de 2021
RESUMEN Objetivo. Analizar la producción investigativa en marketing y su relación con los procesos de exportación en las organizaciones entre 1961 y 2020. Metodología. Se proponen una serie de criterios sobre la producción científica en la temática objeto de estudio, se analizaron diferentes indicadores bibliométricos haciendo uso de las herramientas Bibliometrix (programación estadística R) y VOSviewer, identificando 289 documentos por medio de la base de datos bibliográfica Scopus. Resultados. El estudio permite identificar elementos como los nodos principales y secundarios en la coocurrencia de palabras clave, las fuentes principales de publicación en las temáticas estudiadas, los actores con mayor contribución por país y los artículos de mayor impacto. Conclusiones. La investigación sobre marketing relacionado con procesos de exportación en organizaciones aumenta considerablemente desde el 2000 , una porción minoritaria de la producción total se concentra en investigar las áreas estudiadas en la pequeña y mediana empresa.

PALABRAS CLAVE marketing, investigación, exportaciones, bibliometría, análisis de contenido.

\section{Study on marketing research and its importance in export processes: Bibliometric and thematic analysis in Scopus}

ABSTRACT Objective. To examine marketing research production and its relationship with export processes in organizations between 1961 and 2020. Methodology. Several criteria for scientific production in the subject of study are proposed, and different bibliometric indicators were analyzed with the Bibliometrix (R statistical programming) and VOSviewer tools, yielding 289 documents through the Scopus bibliographic database. Results. The study identified elements such as the main and secondary nodes in the co-occurrence of keywords, the main sources of publication in the topics studied, the authors who contributed the most per country, and the articles with the greatest impact. Conclusions. Since 2000, there has been a significant increase in research on marketing related to export processes in organizations; a minor portion of the total production is focused on researching the areas studied in small and medium-sized enterprises.

KEYWORDS Marketing, research, exports, bibliometrics, content analysis. 


\section{Estudo sobre pesquisa de marketing e sua importância nos processos de exportação: análise bibliométrica e temática no Scopus}

RESUMO Objetivo. Analisar a produção de pesquisa em marketing e sua relação com os processos de exportação nas organizações entre 1961 e 2020. Metodologia. Uma série de critérios é proposta sobre a produção científica no assunto em estudo, diferentes indicadores bibliométricos foram analisados usando as ferramentas Bibliometrix ( $R$ programação estatística) e VOSviewer, identificando 289 documentos por meio da base de dados bibliográficos Scopus. Resultados. 0 estudo permite identificar elementos como os nós principais e secundários na co-ocorrência de palavras-chave, as principais fontes de publicação nos assuntos estudados, os atores com maior contribuição por país e os artigos com maior impacto. Conclusões. A pesquisa de marketing relacionada com os processos de exportação nas organizações aumentou consideravelmente desde 2000 , uma parte minoritária da produção total está concentrada na pesquisa das áreas estudadas nas pequenas e médias empresas.

PALAVRAS CHAVE marketing, pesquisa, exportação, bibliometria, análise de conteúdo. 


\section{Introducción}

El análisis de las publicaciones científicas suele ser una herramienta útil para identificar indicadores relevantes sobre la producción académica en determinada área temática. Autores como Duque y Cervantes (2019) señalan que más allá de los indicadores un análisis detallado de las publicaciones permite ver la evolución de conceptos y prácticas en una disciplina, así como la actualidad en temas críticos que puedan ser pertinentes para organizaciones e investigadores; lo anterior, resulta especialmente interesante en temáticas como marketing y exportaciones que han cobrado relevancia en la última década.

Recientemente Naseramini, Hatami y Hatami (2013) han demostrado que factores como la estrategia de marketing, los canales de divulgación como redes sociales o la cantidad de recursos asignados a las campañas publicitarias pueden tener una influencia directa en el desempeño exportador de una empresa. Esta relación ha despertado el interés de investigadores que hoy dedican mayores esfuerzos a estudiar la relación entre estas temáticas; esto genera una mayor y mejor producción científica que a la vez incentiva el estudio de investigaciones que le preceden, así como la evolución de la producción científica y las tendencias a nivel temático que pueden marcar la investigación de cara al futuro.

Entre los indicadores bibliométricos más frecuentes que se han tenido en cuenta se estudian las principales temáticas que han orientado procesos de investigación que terminan en publicaciones científicas y la distribución de las referencias ordenadas por antigüedad (diferencia entre el año de publicación del artículo o la publicación analizada y las referencias); de esta manera se calcula el número de años, que van desde la actualidad hasta que se publicaron la mitad de las referencias emitidas por la revista en ese año.

En este sentido este documento consta de tres partes: la primera aborda en términos generales los principales referentes teóricos sobre marketing internacional; en la segunda se presenta un análisis bibliométrico de las publicaciones realizadas sobre el tema y finalmente se presentan las conclusiones sobre las publicaciones en marketing y su importancia en los procesos de exportación en las organizaciones.

\section{Marco de referencia}

La globalización económica ha sido un proceso que ha facilitado el desarrollo y crecimiento de los países y en particular de las empresas, las cuales son las encargadas de realizar el intercambio de bienes y servicios. El éxito en este tipo de procesos ha sido estudiado por İpek (2021) para quien depende de la implementación de una serie de estrategias administrativas, operativas, de mercados y financieras en las cuales se hace necesario el relacionamiento y el conocimiento del cliente. De ahí que el marketing se considere una disciplina que facilita el uso de mecanismos para conocer las condiciones en las que opera un mercado y a partir de las cuales se puede obtener mayor posicionamiento (Kotlery Armstrong, 2017; Santesmases, 2012).

Monferrer (2013) considera que el marketing facilita la generación de acciones que mejoran la actividad productiva y comercial de la empresa, trascendiendo con ello en beneficios hacia el cliente.

Para Lozada y Zapata (2015) y Šedík et al. (2018) a través del marketing las empresas pueden mejorar las estrategias, la perspectiva que generen frente al mercado, la promoción y la comunicación, elementos esenciales para prevalecer en el mercado; mientras que Gal et al. (2019) afirman que es una disciplina que vista desde su agilidad facilita la consolidación de la marca, del posicionamiento y de una mejor gestión en los procesos realizados en la empresa.

A lo largo de los últimos años se han realizado diversos estudios sobre marketing (Martínez-López et al., 2018) en los que se denota su importancia para el crecimiento económico. Es importante resaltar que este no puede ser considerado solo como mecanismo de venta o comercialización de productos o servicios sino que sus conceptos facilitan la identificación de necesidades presentes en el mercado (Schnarch, 2017; Morgan et al., 2019). 
Por ello las estrategias que se generen dependen del conocimiento de todos los actores que participen en el proceso (Mediano y Beristain, 2015), teniendo en cuenta que los mercados evolucionan y sus características se someten a diversos factores (tales como ubicación, aspectos demográficos, sociales, culturales, económicos, políticos) (Wilson-Barnao, 2017; Peral, Gaitán and Jerónimo, 2013; Vásquez, 2004). Dicha situación lleva a las empresas a tener en cuenta aspectos esenciales para comercializar sus productos, tomando en cuenta las características de cada mercado y de los actores que participan en él.

Así las cosas, en respuesta al proceso de globalización de la economía, surge el marketing internacional a través del cual las empresas pueden responder a las tendencias de los mercados y determinar los elementos que utilizarán para promocionar sus productos y/o servicios (Jiménez, 2011).

Pauly Mas (2020) plantean lo importante que es para una empresa conocer los aspectos que caracterizan a un mercado. Esto se fundamenta en los cambios impulsados por los avances tecnológicos, geográficos y demográficos movidos por las tendencias que se plantean en el mercado que hacen que los consumidores vean los productos y necesidades a satisfacer de otra manera (Vrontis et al., 2020; Katsikeas, Leonidou and Zeriti, 2019).

El marketing internacional se enfoca en el desarrollo de actividades que llevan a la incursión en mercados externos (Morgan, Feng and Whitler, 2018), al uso de sistemas como la Internet y las redes sociales que facilitan la comunicación empresarial y su relacionamiento con el cliente (Samiee, 2020; Sheth, 2020; Prasad, Ramamurthy and Naidu, 2001; Bianchi and Mathews, 2016) y a la incorporación de procesos innovadores (Anning-Dorson, 2018; Efrat, Gilboa and Yonatany, 2017).

De ahí que Leonidou et al. (2018) señalen que existe un reconocimiento especial por esta disciplina, llevando a que se realicen investigaciones en las que el marketing internacional se convierte en el centro de diferentes elementos determinantes para el crecimiento y desarrollo empresarial.

Autores como Valenzuela et al. (2017) han desarrollado estudios y análisis bibliométricos en los que presentan las principales tendencias del marketing. Otros como Foreman y Money (1995), además de hacer referencia a su concepto y características, destacan los elementos que son clave para su medición interna.

Su contribución fundamenta este estudio especialmente porque permite conocer los elementos y fundamentos para el marketing internacional, las tendencias, las principales revistas que han generado contribuciones sobre el tema y su impacto en términos de aporte al conocimiento. Teniendo en cuenta que - a partir del marketing, sus conceptos, factores, herramientas y campos de acción - aportan significativamente a la generación de estrategias para el crecimiento de la economía (Cavusgil and Nevin, 1981; Magnusson et al., 2013). De ahí que uno de los temas que favorece significativamente la conexión entre marketing e internacionalización sean las exportaciones.

Según Rekarti, Doktoralina y Saluy (2018) el marketing se convierte en un elemento esencial que promueve el desarrollo comercial, en especial de las exportaciones en las empresas que quieren incursionar en mercados globalizados como es el caso de las pequeñas y medianas empresas.

Por ello la incursión en nuevos mercados y el desarrollo de nuevos negocios han generado el incremento en el número de empresas que comienzan a exportar (Castro-González etal., 2016) y como consecuencia requieren del marketing internacional para identificar oportunidades, analizar las necesidades existentes, conocer la competencia y a partir de allí saber cómo abordar una estrategia (Ruzo et al., 2011; Morgan, Katsikeas and Vorhies, 2012; Mac and Evangelista, 2016).

Así pues, Diamantopoulos, Schlegelmilch y Tse (1993) afirman que resulta importante incluir la investigación de procesos de comercialización para la generación de una estrategia de marketing. Esto facilita la comprensión de la dinámica existente y provee elementos que permitirán un mejor relacionamiento entre los actores que participan en el mercado, enfoque que enriquece los objetivos establecidos por la empresa (Lages and Montgomery, 2004).

De esta manera son diversos los casos que se pueden presentar en los cuales existe una influencia del marketing internacional en el desempeño de 
las exportaciones (Samiee and Chirapanda, 2019), así como el desarrollo de modelos que surgen para mejorar la actividad comercial (Umboh and Aryanto, 2019). Algunos de estos resultados que pueden ser vistos desde la mezcla de marketing, el desarrollo de la estrategia y planeación y la ejecución del proceso (Ogunmokun and Ng, 2004). En cuanto a referentes en análisis bibliométricos aplicados a estas disciplinas autores como Norris y Oppenheim (2007) resaltan que gracias a la mayor disponibilidad de herramientas gratuitas (como, por ejemplo, Gephi, Leximancer, VOSviewer) este tipo de investigaciones son más frecuentes.

Hoy no existen investigaciones que combinen específicamente a las temáticas que aborda este artículo, aunque hay estudios bibliométricos para áreas determinadas del marketing. Salimi, Tavassoli y Gilani (2019) realizan la estimación de indicadores específicos sobre el impacto de las redes sociales en el marketing, mientras que Valencia et al. (2019) relacionan el marketing con la industria creativa; recientemente OsorioAndrade, Murcia-Zorrilla y Arango-Espinal (2020) han aplicado estudios bibliométricos sobre temas coyunturales en la actualidad de la disciplina como el city marketing, aunque ninguno de los estudios mencionados involucra la relación con los procesos de exportación.

\section{Metodología}

Para la evaluación de la producción científica por medio de indicadores bibliométricos, Bordons (1999) resalta la importancia de seleccionar adecuadamente una base de datos que cuente con reconocimiento en la comunidad académica y que contenga a la vez un alto volumen de publicaciones sobre la materia que se desea investigar. Como herramienta, el análisis bibliométrico ofrece la posibilidad de detallar la evolución histórica de la influencia y producción en relación a la importancia del marketing en los procesos de exportación de la empresa privada.

Inicialmente se contempló aplicar el análisis bibliométrico sobre los resultados obtenidos en los portales de Web of Science (WoS), Scopus y Google Scholar que hoy son tres de las bases de datos más importantes en materia de publicaciones científicas multidisciplinares. Según Cadavid, Awad y Franco (2012) estas son en esencia las que por su estructura, calidad de los documentos y capacidad tecnológica son más útiles a la hora de realizar análisis bibliométricos. De estas tres bases de datos se decidió utilizar Scopus debido a las herramientas que permitían aplicar un filtro más riguroso a las publicaciones consultadas, adicionalmente se ajusta mejor a los objetivos planteados en el estudio.

Es importante señalar que para la selección también fueron relevantes los resultados de la investigación de Granda-Orive et al. (2013) que reconoce que la base de datos Scopus presenta ventajas como una interfaz más amigable con el usuario, acceso a documentos revisados por pares, toma en cuenta el cálculo del factor $\mathrm{H}$ - valorando así el recorrido e influencia de los investigadorese incluye la totalidad de lo indexado en otras bases de datos tales como Medline, Embase y Compendex; algunas de estas características se encuentran limitadas en las otras bases de datos, por lo que no se podría hacer un trabajo comparativo y unificación de resultados.

Una vez seleccionada la base de datos se definieron los criterios y ecuación de búsqueda centrada en los dos conceptos principales de estudio que corresponden a marketing y exportaciones, a nivel práctico en la base de datos la ecuación aplicada fue: «(TITLE [Marketing] AND TITLE [Exports])».

Como resultado de la búsqueda se obtuvo coincidencia de 289 resultados, correspondientes a diferentes productos resultados de procesos de investigación, principalmente artículos científicos publicados en revistas indexadas; en cuanto a los años tenidos en cuenta se ha considerado un horizonte temporal de publicaciones desde 1961 hasta 2020; sobre esta muestra se ha realizado la estimación de indicadores especializados en estudios bibliométricos propuestos por Naveen et al. (2020), así entre las diferentes categorías existentes se aplicaron los indicadores: coocurrencia de palabras clave; volumen de producción por año; citación por revista; artículos con mayor citación; tipología de producción y volumen de publicación por país. La herramienta principal para el procesamiento de los resultados fue la programación estadística R, Bibliometrix, 
específicamente la base de datos fue procesada en la aplicación Biblioshiny.

\section{Resultados y discusión}

Los resultados sobre la coocurrencia entre palabras clave de la muestra seleccionada presentan una concentración principal en la palabra 'marketing' seguida de 'exportaciones' o "comercio internacional", lo cual se deriva de ser las temáticas principales de estudio (figura 1); la coocurrencia de palabras como 'China', "Estados Unidos” y 'Australia' puede dar una idea sobre los mercados principales que se han estudiado; mientras que la concentración en palabras como 'agricultura' e "industria farmacéutica" indican sectores económicos particulares sobre los que se han concentrado los estudios analizados.

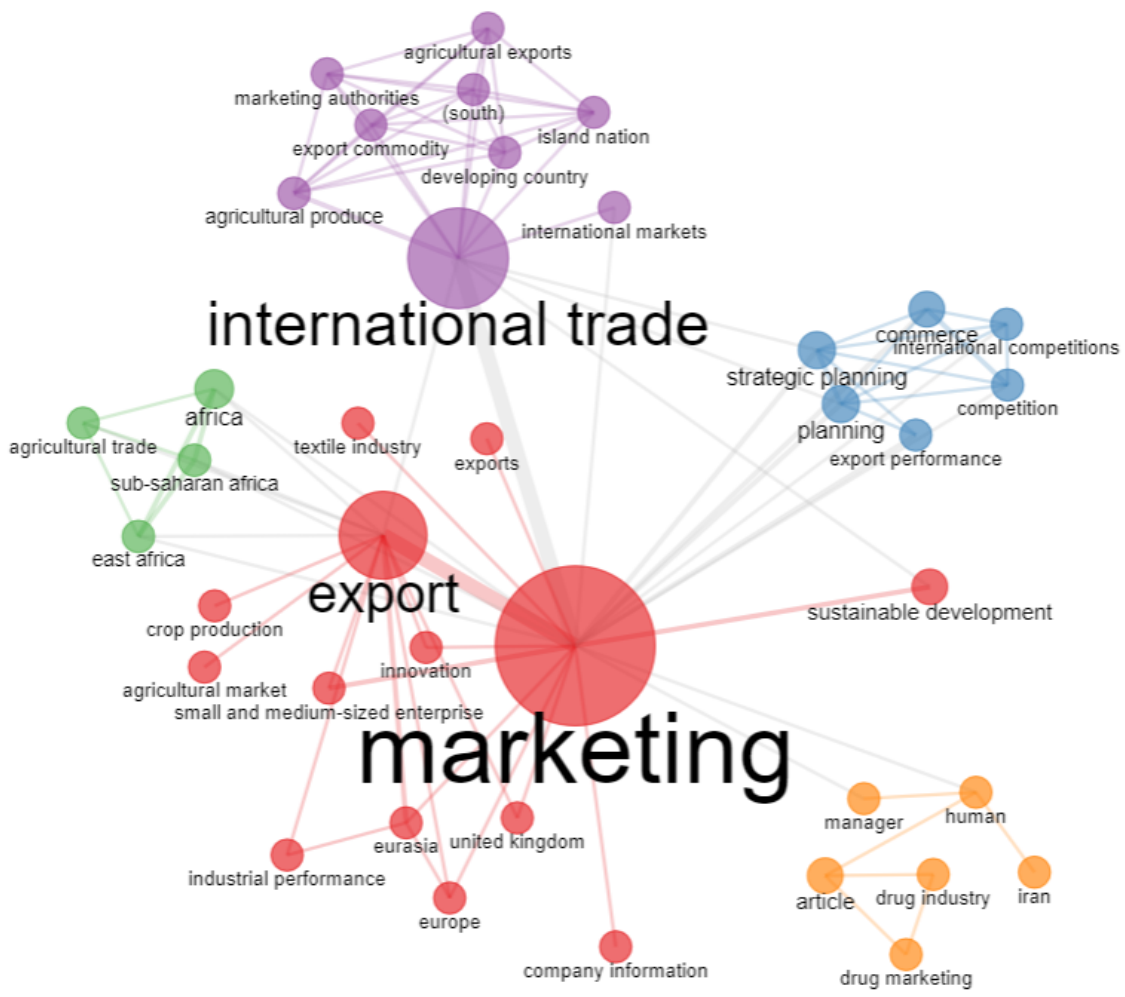

Figura 1. Coocurrencia entre palabras clave. Fuente: elaboración propia por parte de los autores.

Otras palabras clave tales como comercio, economía, competitividad y manufactura aparecen con menor coocurrencia; aunque estas reflejan la investigación en temas relacionados con las actividades que promueven análisis de las condiciones que se presentan en los mercados, los sectores y las empresas que lo integran y de los elementos que promueven el desarrollo, crecimiento y competitividad de dichos procesos.
Respecto al volumen de publicaciones entre 1961 y 2020 los documentos relacionados con 'marketing' y 'exportaciones' pasaron de 1 publicación anual (en promedio) para los primeros años estudiados a una media de 17 documentos en la última década (figura 2), lo cual refleja un incremento considerable en los procesos de investigación sobre estas dos temáticas. 


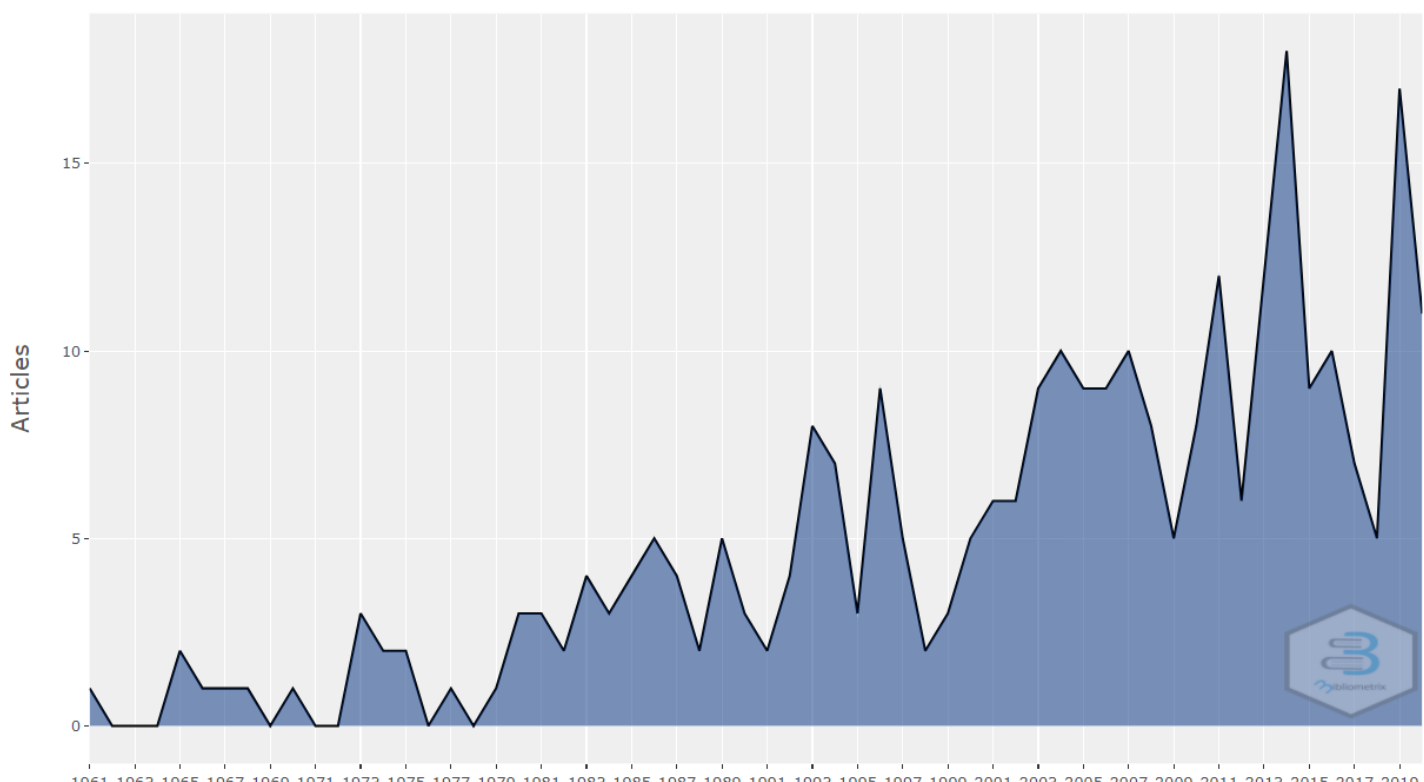

Figura 2. Evolución del volumen anual de publicaciones. Fuente: elaboración propia por parte de los autores.

El período que mayor concentra la publicación académica se dio entre 2014 a 2020, alcanzando un total de 70 documentos; esto representa un 24,22\% del total de la producción científica estudiada; este incremento puede ser influenciado por elementos tales como los avances tecnológicos en temas de comercio electrónico, herramientas digitales para el marketing, un número mayor de casos para estudio y el fortalecimiento de la logística internacional.

En cuanto a los diferentes tipos de productos estudiados para el horizonte de tiempo evaluado hay una concentración del $74 \%$ en artículos científicos publicados en revistas indexadas, otros artículos de revisión o reflexión tienen una participación del $6 \%$, capítulos de libro con un $5 \%$ y la producción restante se puede clasificar en productos de otra naturaleza (tabla 1); este comportamiento refleja que la producción se concentra en productos de un mayor nivel investigativo.

Tabla 1. Tipología de documentos publicados

\begin{tabular}{|c|c|}
\hline Tipo de documento & Total \\
\hline Article & 213 \\
\hline Review & 20 \\
\hline Book Chapter & 16 \\
\hline
\end{tabular}

\begin{tabular}{lr}
\hline \multicolumn{1}{c}{ Tipo de documento } & Total \\
\hline No definido & 14 \\
\hline Conference Paper & 13 \\
\hline Business Article & 5 \\
\hline Book & 2 \\
\hline Short Survey & 2 \\
\hline Note & 1 \\
\hline Letter & 1 \\
\hline Total & $\mathbf{2 8 9}$ \\
\hline
\end{tabular}

Fuente: elaboración propia por parte de los autores.

Un análisis sobre los resultados de la producción por país evidencia que la investigación en estas temáticas se realiza principalmente en países como Estados Unidos, Reino Unido, Australia, España, China e Indonesia (figura 3), de los países de la región destaca únicamente Brasil con una contribución discreta de 7 documentos en el total de producción histórica en las temáticas analizadas. En cuanto a los idiomas utilizados para la publicación de documentos se tiene el inglés, alemán y en menor medida el español. 


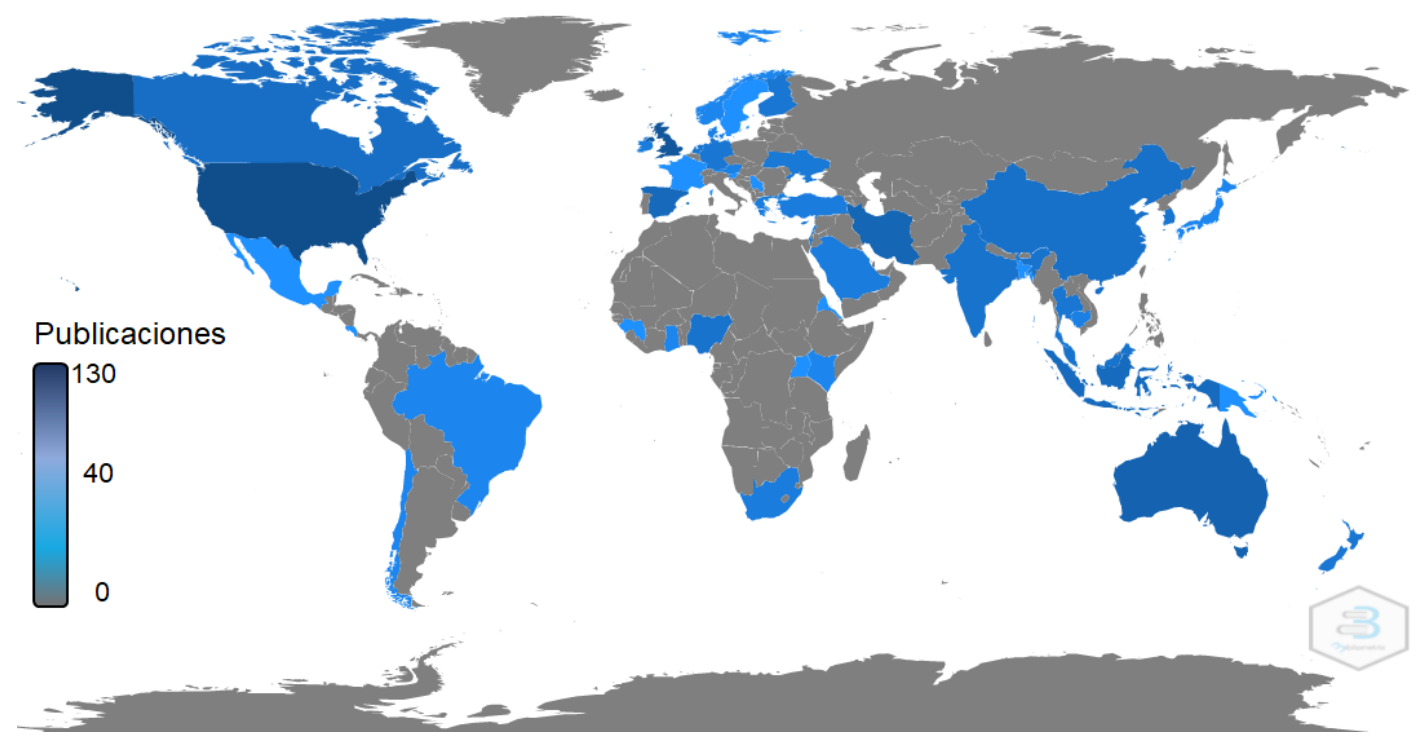

Figura 3. Volumen producción por país. Fuente: elaboración propia por parte de los autores.

Una observación a las fuentes de mayor relevancia desde la perspectiva de citación por revista permite evidenciar que de la muestra estudiada los documentos son publicados principalmente en revistas norteamericanas y europeas, destaca la alta contribución de de la revista Journal of Marketing de la American Marketing Association y la revista Journal of
International Studies del Centro para la investigación Sociológica con un total de 607 y 574 citaciones respectivamente. En cuanto al volumen de citación las 15 revistas de mayor consulta consolidan el $95 \%$ de las citaciones (figura 4), lo cual presenta una alta concentración respecto al universo total de fuentes en las que se han publicado estudios sobre marketing y exportaciones.

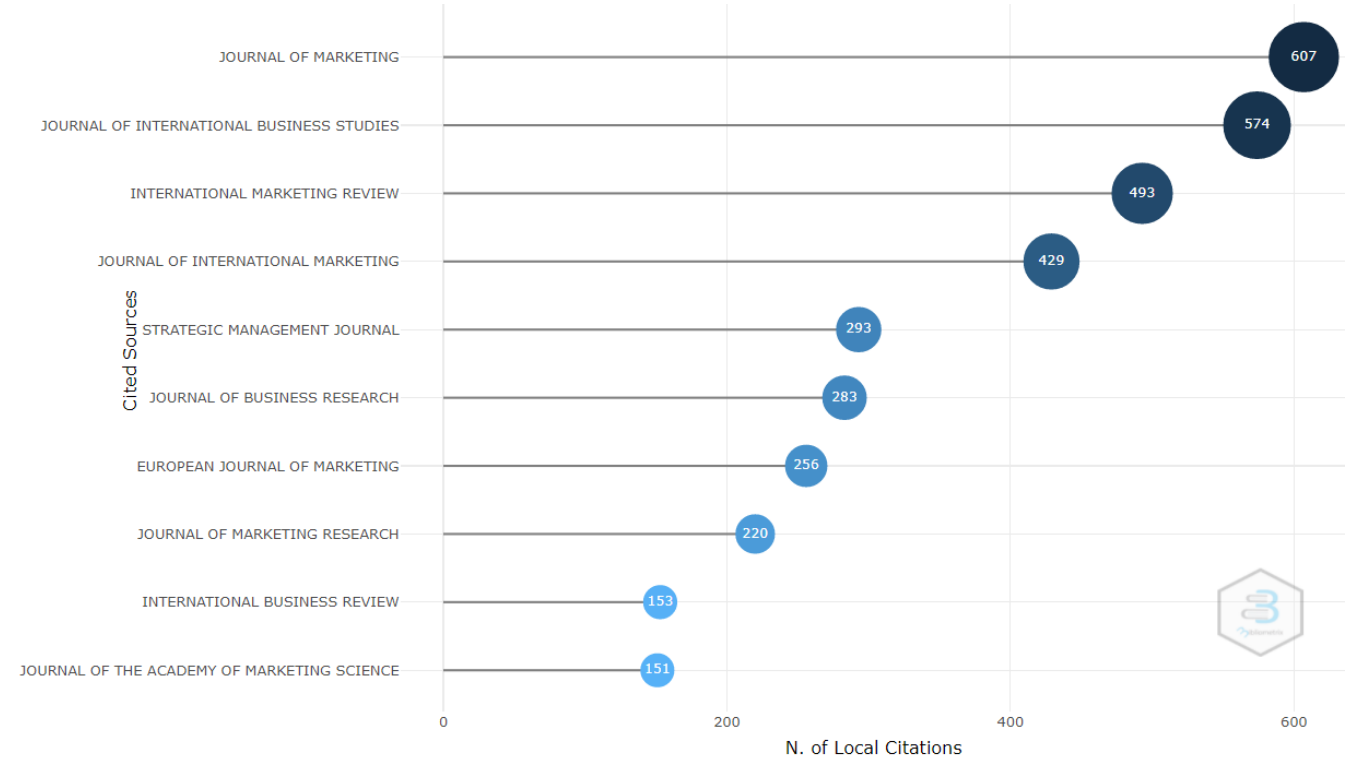

Figura 4. Fuentes de mayor relevancia. Fuente: elaboración propia por parte de los autores. 
Una revisión más detallada al volumen de citación por artículos específicospermite realizar una aproximación a los documentos de mayor consulta y relevancia académica respecto al marketing y su importancia en procesos de exportación; para el horizonte de tiempo estudiado el articulo con mayor volumen de citación asciende a un total de 380 y corresponde al estudio titulado: "Marketing strategy determinants of export performance: A metaanalysis"; este es seguido de otros 10 documentos que agrupan un $65 \%$ de la citación total de los documentos estudiados (tabla 2).

Tabla 2. Artículos con mayor número de citaciones

\begin{tabular}{|c|c|c|}
\hline Titulo del artículo & País & Citas \\
\hline Marketing strategy determinants of export performance: A meta-analysis & Chipre & 380 \\
\hline $\begin{array}{l}\text { Market orientation and performance of export ventures: The process through marketing } \\
\text { capabilities and competitive advantages }\end{array}$ & Estados Unidos & 250 \\
\hline Successful Export Marketing Management: Some Empirical Evidence & Dinamarca & 245 \\
\hline Firm and management characteristics as discriminators of export marketing activity & Estados Unidos & 243 \\
\hline $\begin{array}{l}\text { Export marketing strategy implementation, export marketing capabilities, and export venture } \\
\text { performance }\end{array}$ & Estados Unidos & 197 \\
\hline $\begin{array}{l}\text { Experiential and Informational Knowledge, Architectural Marketing Capabilities, and the Adaptive } \\
\text { Performance of Export Ventures: A Cross-National Study }\end{array}$ & Estados Unidos & 183 \\
\hline $\begin{array}{l}\text { The Effect of Export Marketing Capabilities on Export Performance: An Investigation of Chinese } \\
\text { Exporters }\end{array}$ & Estados Unidos & 169 \\
\hline $\begin{array}{l}\text { The influence of internet-marketing integration on marketing competencies and export } \\
\text { performance }\end{array}$ & $\begin{array}{l}\text { No definido en } \\
\text { Scopus }\end{array}$ & 148 \\
\hline The first export order: A marketing innovation & Reino Unido & 146 \\
\hline Total de citas & & 2107 \\
\hline
\end{tabular}

Fuente: elaboración propia por parte de los autores.

Finalmente es importante destacar que la mayor parte de estos artículos consolidan información en la cual se hace referencia a la relación existente entre el marketing y las exportaciones y la importancia que representa para el crecimiento de la economía, de las empresas y de los sectores.

\section{Conclusiones}

A pesar de que históricamente el marketing y las exportaciones son temáticas que han mantenido una relación a nivel de publicaciones científicas, antes del 2000 el volumen de producción era discreto sin exceder las 20 publicaciones anuales; sin embargo se evidencia que desde 2003 las publicaciones aumentaron sustancialmente al incrementarse en aproximadamente en 1,43 veces promedio cada año, lo cual es un signo positivo de cara al futuro en el estudio de estas temáticas.

Existe la necesidad de que las temáticas se estudien con mayor recurrencia y profundidad por autores y centros de investigación en economías no desarrolladas o emergentes, esto debido a que menos del $10 \%$ de la producción total estudiada durante la investigación ha sido aportada por autores de habla hispana o con estudios de caso de compañías regionales.

Hay una alta concentración en los estudios temáticos orientados a evaluar la relación, 
incidencia o impacto del marketing en el rendimiento exportador enfocado únicamente a compañías multinacionales y de gran reconocimiento; si bien es cierto que es en este tipo de organizaciones en las que se concentra el mayor volumen de operaciones de exportación, también puede ser muy beneficioso estudiar variables y fenómenos alrededor de las temáticas en las pequeñas y medianas empresas.

A pesar de que en los últimos años estudiados el volumen de publicaciones ha disminuido, los autores estudiados resaltan la creciente importancia del marketing en los procesos de exportación en las organizaciones; en gran medida esto puede deberse al mayor acceso a la información y medios masivos por parte de la población en general, mayores canales de difusión de información y finalmente sistemas económicos enfocados a facilitar el comercio exterior.

\section{Referencias}

Anning-Dorson, T. (2018). Innovation and competitive advantage creation. International Marketing Review, 35(4), 580-600.

Bianchi, C. and Mathews, S. (2016). Internet marketing and export market growth in Chile. Journal of Business Research, 69(2), 426-434.

Bordons, M. (1999). Evaluación de la actividad científica a través de indicadores bibliométricos. Revista Española de Cardiología, 52(10), 790800 .

Cadavid, L., Awad, G. y Franco, C. (2012). Análisis bibliométrico del campo modelado de difusión de innovaciones. Estudios Gerenciales, 28(edición especial), 213-236.

Castro-González, P. et al. (2016). Export intensity and strengths perceived in the marketing strategy: A perspective based on Spanish export companies' experience. Intangible Capital, 12(5), 1484-1504.

Cavusgil, S.T. and Nevin, J.R. (1981). Internal determinants of export marketing behavior:
An empirical investigation.Journal of Marketing Research, 18(1), 114-119.

Diamantopoulos, A., Schlegelmilch, B.B. and Tse, K.Y. (1993). Understanding the role of export marketing assistance: empirical evidence and research needs. European Journal of Marketing, 27(4), 5-18.

Efrat, K., Gilboa, S. and Yonatany, M. (2017). When marketing and innovation interact: The case of born-global firms. International Business Review, 26(2), 380-390.

Duque, P.y Cervantes, L.S. (2019). Responsabilidad social universitaria: una revisión sistemática y análisis bibliométrico. Estudios Gerenciales, 35(153), 451-464.

Foreman, S.K. and Money, A.H. (1995). Internal marketing: concepts, measurement and application. Journal of Marketing Management, 11(8), 755-768.

Gal, D. et al. (2019). Marketing Agility: Conceptualization, Research Propositions, and a Research Agenda. Kenan Institute of Private Enterprise Research Paper, 19-22.

Granda-Orive, J.I. et al. (2013). Ciertas ventajas de Scopus sobre Web of Science en un análisis bibliométrico sobre tabaquismo. Revista Española de Documentación Científica, 36, 2, 1-11.

Jiménez, A. (2011). La competitividad como herramienta empresarial necesaria para la inserción de las empresas en los mercados globales. Revista Venezolana de Análisis de Coyuntura, 17(1), 91-114.

İpek, İ. (2021). The relevance of international marketing strategy to emerging-market exporting firms: from a systematic review towards a conceptual framework. International Marketing Review, 38(2), 205-248.

Katsikeas, C., Leonidou, L. and Zeriti, A. (2019). Revisiting international marketing strategy in a digital era. International Marketing Review, $37(3), 405-424$. 
Kotler, P. y Armstrong, G. (2002). Fundamentos de marketing. Naucalpan de Juárez, México: Pearson PLC.

Lages, L.F. and Montgomery, D.B. (2004). Export performance as an antecedent of export commitment and marketing strategy adaptation. European Journal of Marketing, 38(9), 1186-1214.

Leonidou, L.C. etal. (2018). International marketing research: A state-of-the-art review and the way forward. En Advances in Global Marketing: A Research Anthology (pp. 3-33). New York, USA: Springer.

Lozada, F. and Zapata, M. (2016). What is Marketing? A Study on Marketing Managers' Perception of the Definition of Marketing. Forum Empresarial, 21(1), 49-69.

Mac, L. and Evangelista, F. (2016). The relative impact of market orientation and entrepreneurship on export performance: do we really know enough? Journal of Global Marketing, 29(5), 266-281.

Magnusson, P. et al. (2013). The role of cultural intelligence in marketing adaptation and export performance. Journal of International Marketing, 21(4), 44-61.

Martínez-López, F.J. et al. (2018). Fifty years of the European Journal of Marketing: a bibliometric analysis. European Journal of Marketing, 52(1), 439-468.

Mediano, L.y Beristain, J. (2014). Marketing práctico para pequeños negocios. Madrid, España: Ediciones Pirámide.

Monferrer, D. (2013). Fundamentos de marketing. Castellón de la Plana, España: Universitat Jaume I.

Morgan, N.A. et al. (2019). Research in marketing strategy. Journal of the Academy of Marketing Science, 47(1), 4-29.

Morgan, N.A., Katsikeas, C.S. and Vorhies, D.W. (2012). Export marketing strategy implementation, export marketing capabilities, and export venture performance. Journal of the Academy of Marketing Science, 40(2), 271-289.

Morgan, N.A., Feng, H. and Whitler, K.A. (2018). Marketing capabilities in international marketing. Journal of International Marketing, 26(1), 61-95.

Naseramini, S., Hatami, Z. and Hatami, A. (2013). Impact of export marketing strategy on export performance (Case Study: Tabriz). International Journal of Management and Humanity Sciences, 2(6), 521-529.

Norris, M. and Oppenheim, C. (2007). Comparing alternatives to the Web of Science for coverage of the social sciences' literature. Journal of Informetrics, 1(2), 161-169.

Ogunmokun, G. and Ng, S. (2004). Factorsinfluencing exportperformance in international marketing: A study of Australian firms. International Journal of Management, 21(2), 172.

Osorio-Andrade, C., Murcia-Zorrilla, C. and ArangoEspinal, E. (2020). City marketing research: a bibliometric analysis. Revista Escuela de Administración de Negocios, 89(1), 1-28.

Paul, J. and Mas, E. (2020). Toward a 7-P framework for international marketing. Journal of Strategic Marketing, 28(8), 681-701.

Peral, B.P., Gaitan, J.A. and Jeronimo, M.A.R. (2013). The role of socio-demographic variables in the use of internet-based applications by older people. Innovar, 23(48), 55-66.

Prasad, V.K., Ramamurthy, K. and Naidu, G.M. (2001). The influence of Internet-marketing integration on marketing competencies and export performance. Journal of International Marketing, 9(4), 82-110.

Rekarti, E., Doktoralina, C.M. and Saluy, A.B. (2018). Development model of marketing capabilities and export performance of SMEs: A proposed study. European Journal of Business and Management, 10(12), 107-114. 
Ruzo, E. et al. (2011). Resources and international marketing strategy in exportfirms. Management Research Review, 34(5), 496-518.

Salimi, D., Tavassoli, K. and Gilani, A. (2019). The impact of social media on marketing using bibliometrics analysis. International Journal of Data and Network Science, 1(1), 165-184.

Samiee, S. and Chirapanda, S. (2019). International marketing strategy in emerging-market exporting firms. Journal of International Marketing, 27(1), 20-37.

Samiee, S. (2020). International marketing and the internet: a research overview and the path forward. International Marketing Review,37(3), 425-436.

Santesmases, M. (2012). Marketing. Madrid, España: Ediciones Pirámide.

Šedík, P. et al. (2018). Generation marketing in strategic marketing management: case study of honey market. Polish Journal of Management Studies, 18(1), 326-337.

Schnarch, A. (2017). Marketing de fidelización. Bogotá, Colombia: ECOE Ediciones.

Sheth, J.N. (2020). Borderless Media: Rethinking International Marketing. Journal of International Marketing, 28(1), 3-12.

Umboh, I.A. and Aryanto, V.D.W. (2019). Improving Export Marketing Performance: A Conceptual Model of Adaptation Marketing Strategy. Quality Access to Success, 20(171), 62-69.

Valencia, J. et al. (2019). Evolución y tendencias investigativas en marketing orientado a industrias culturales y creativas: un análisis bibliométrico en Scopus. Semestre Económico, 22(53), 189-213.

Valenzuela, L.M. et al. (2017). Thirty years of the Journal of Business \& Industrial Marketing: A bibliometric analysis. Journal of Business \& Industrial Marketing, 32(1), 1-17.

Vásquez, J. (2004). Pasado, presente y futuro de las dimensiones pública y social en el desarrollo conceptual del marketing. International Review on Public and Nonprofit Marketing, 1(1), 9-34.

Vrontis, D. etal. (2020). Cause-related marketing in international business: what works and what does not? International Marketing Review, 37(4), 593-601.

Wilson-Barnao, C. (2017). How algorithmic cultural recommendation influence the marketing of cultural collections. Consumption Markets \& Culture, 20(6), 559-574. 